Ríos de Deus, M.P. (2014). Sistemas de garantía de calidad en centros de Educación Superior. Revista Electrónica Interuniversitaria de Formación del Profesorado, 17 (3), 17-34.

DOI: http://dx.doi.org/10.6018/reifop.17.3.204021

\title{
Sistemas de garantía de calidad en centros de Educación Superior
}

Ma Paula Ríos de Deus

Técnico de Programas de la Agencia para la Calidad del Sistema Universitario de Galicia

\section{Resumen}

La preocupación de las universidades por la calidad institucional y los cambios en la legislación española han sido el punto de partida para el diseño e implantación de Sistemas de Garantía de Calidad (SGC) en los centros de las universidades españolas, a través del Programa AUDIT.

AUDIT ha sido diseñado, siguiendo las directrices de ENQA (European Association for Quality Assurance in Higher Education) con el objetivo de cubrir todas las dimensiones y los grupos de interés involucrados en el proceso formativo, por la "Axencia para a Calidade do Sistema Universitario de Galicia" (ACSUG), la "Agencia Nacional de Evaluación de la Calidad y Acreditación" (ANECA) y la "Agència per a la Qualitat del Sistema Universitari de Catalunya" (AQU Catalunya), posteriormente se incorporó la "Euskal Unibertsitate Sistemaren Kalitate Agentzia" (UNIBASQ). Se presenta oficialmente a las universidades en 2007 para facilitar las orientaciones para el diseño, desarrollo e implementación de los SGC y poner en práctica procedimientos de reconocimiento del diseño y certificación de la implantación de los mismos.

Es muy destacable el elevado grado de participación de las universidades en AUDIT. Entre 2007 y 2013 participaron en la evaluación del diseño de los SGC 62 universidades españolas $(80,5 \%)$. Actualmente se ha comenzado la fase de certificación de la implantación.

\section{Palabras clave}

Calidad institucional; diseño de SGC; implantación de SGC; mejora continua. 


\title{
Quality assurance systems in higher education institutions
}

\author{
Abstract \\ The concern of the universities for the institutional quality and changes in Spanish \\ legislation have been the starting point for the design and implementation of quality \\ assurance systems (QAS) in higher education institutions, through the AUDIT Program.
}

AUDIT has been designed, following the guidelines of ENQA (European Association for Quality Assurance in Higher Education) with the aim of covering all dimensions and interest groups involved in the learning process, by the "Axencia para a Calidade do Sistema Universitario de Galicia" (ACSUG), the "Agencia Nacional de Evaluación de la Calidad y Acreditación" (ANECA) and the "Agència per a la Qualitat del Sistema Universitari de Catalunya" (AQU Catalunya), subsequently joined the "Euskal Unibertsitate Sistemaren Kalitate Agentzia" (UNIBASQ). Officially is presented to universities in 2007 to provide guidelines for the design, development and implementation of the QAS and implement procedures of recognition of the design and certification of the implementation.

Very remarkable is the high degree of participation of universities in AUDIT. Between 2007 and 2013 participated in the evaluation of the design of the QAS 62 Spanish universities $(80,5 \%)$. Currently, the certification of the implementation has begun.

\section{Key words}

Institutional quality; QAS design; QAS implementation; continuous improvement.

\section{Introducción}

La reformada ley española de universidades (Ley Orgánica 4/2007 por la que se modifica la Ley Orgánica 6/2001, de Universidades) recoge la necesidad de establecer criterios y normas de garantía de calidad como elementos esenciales en el diseño de los nuevos títulos oficiales y los considera como un objetivo esencial de la política universitaria.

El Real Decreto 1393/2007, de 29 de octubre, por el que se establece la ordenación de las enseñanzas universitarias oficiales , determina que "Ios Sistemas de Garantía de la Calidad, (...) son el fundamento para que la nueva organización de las enseñanzas funcione eficientemente y para crear la confianza sobre la que descansa el proceso de acreditación de los títulos", así como que toda "propuesta de título debe incluir un Sistema de Garantía de Calidad que asegure el control, la revisión y mejora continua del mismo".

Como consecuencia de estas reformas legislativas, la nueva estructura organizativa de los estudios universitarios en España establece la garantía de la calidad como uno de los elementos básicos que deben incorporar los programas educativos y que es esencial en el diseño de todos los títulos.

La utilización de estrategias de mejora continua no es una novedad en las universidades españolas. Con el apoyo de las agencias de evaluación, desde finales de la pasada década, las universidades desarrollan procesos de evaluación y certificación de sus enseñanzas y recogen las opiniones de estudiantes y otros colectivos:

- Desde 1981 se ha recogido, a través de encuestas, información sobre la satisfacción de los estudiantes universitarios en relación con la enseñanza que recibían, 
incorporando, posteriormente (entre 2006-2008), otros grupos de interés: docentes, graduados, personal de administración y empleadores.

- Entre 1996 y 2006, la garantía de calidad en las universidades españolas estaba asociada a procesos voluntarios de evaluación institucional, desarrollados a través del Plan Nacional de Evaluación de la Calidad (PNECU 1996-2000), del II Plan de Calidad de las Universidades (II PCU 2001-2003) y del Programa de Evaluación Institucional (PEI 2004-2006). Estos programas incluían un proceso de autoevaluación (una comisión constituida por representantes de los grupos de interés internos al centro elaboraban un informe sobre la calidad de la enseñanza, la investigación y la gestión) y un proceso de evaluación externa con visita, gestionado por las agencias de calidad y llevado a cabo por comisiones de evaluación externa.

- En 2001, dentro del II PCU, se desarrolló el proceso de evaluación de los servicios universitarios (biblioteca, relaciones internacionales y otros servicios de apoyo a los estudiantes). Dicho proceso estaba centrado en la gestión de la calidad y la excelencia de la organización.

- Paralelamente, diversas universidades llevaron a cabo procesos de evaluación de los servicios a través de modelos de reconocimiento internacional, tales como el Modelo de excelencia EFQM y las normas UNE-EN ISO 9001.

- Desde 2001, la "Axencia para a Calidade do Sistema Universitario de Galicia ACSUG" realiza análisis de inserción laboral de los titulados en las tres universidades del Sistema Universitario de Galicia, enviando los resultados obtenidos a las tres universidades y a todos sus centros. Con la finalidad de que estos resultados contribuyan al diseño de estrategias para la revisión y mejora de la calidad de la oferta formativa.

Ahora bien, un análisis de estas iniciativas, realizado a la luz de las exigencias del Espacio Europeo de Educación Superior (EEES), revela que las universidades necesitan un planteamiento ordenado, estructurado y sistemático respecto a la mejora continua. El diseño e implementación de procedimientos de garantía de calidad aporta un nuevo sentido a las diferentes actuaciones emprendidas en materia de evaluación de enseñanzas, servicios y profesorado, de análisis de la satisfacción e inserción laboral o de información a la sociedad.

El sistema universitario español en su conjunto y cada una de las instituciones universitarias han de responder al compromiso de satisfacción de las necesidades y expectativas generadas por la sociedad. En el contexto de una sociedad del conocimiento y de creciente internacionalización de la oferta universitaria, asegurar la calidad de la formación en los diferentes estudios es un reto ineludible, máxime cuando la sociedad ha depositado la confianza y ha aportado recursos considerables para que las universidades cumplan con dicha función.

En correspondencia a la confianza que la sociedad deposita en la gestión autónoma de las universidades y a la transparencia exigida en el marco del EEES, estas deben garantizar que sus actuaciones están en la dirección apropiada para lograr los objetivos asociados a las enseñanzas que imparten. Para ello, las universidades deben contar con políticas y Sistemas de Garantía de Calidad (SGC) formalmente implantados y públicamente disponibles.

\section{Implantación de SGC}

La preocupación de las universidades por la calidad institucional y estos cambios en la legislación española han sido el punto de partida para el diseño e implantación de SGC en 
los centros, a través del Programa AUDIT. Este programa da apoyo a la necesidad requerida por las universidades de disponer de procedimientos asociados a la garantía de calidad y dotarse de mecanismos formales para la aprobación, control, revisión periódica y mejora de sus títulos, así como de facilitar la transición de las enseñanzas universitarias y su adaptación al EEES.

Por otra parte, nuestro marco normativo también señala que las agencias de evaluación han de contribuir, a través de los procesos de evaluación externa, a garantizar los objetivos de calidad universitaria. La conjunción ordenada de las actuaciones internas y externas de las universidades y las agencias ha de constituir el sistema de garantía de calidad del Sistema Universitario Español (SUE). De aquí que, no sólo las universidades, sino también las agencias deben establecer sus propios SGC, así como someterse a la evaluación externa de acuerdo a criterios y directrices europeas adoptados en Bergen (2005) por los ministros firmantes del Proceso de Bolonia.

El Programa AUDIT ${ }^{1}$ motiva a los centros a reflexionar sobre los cambios que son necesarios incorporar para integrar, de un modo sistemático, los procesos de mejora continua en un sistema de garantía de calidad y facilitar la necesidad de la rendición de cuentas a la sociedad, con el respeto de su autonomía, atendiendo a su diversidad y promoviendo la creatividad e innovación en cada uno de ellas; integrando así, todas las actividades, que hasta el momento, se venían desarrollando y que estaban relacionadas con la garantía de calidad de la enseñanza. La implementación de un SGC favorece el proceso de verificación, seguimiento y renovación de la acreditación de los títulos oficiales.

El objetivo del programa AUDITes orientar a las universidades españolas en el proceso de definición, diseño e implementación efectiva del SGC. En este sentido, el programa está destinado a servir como un marco de referencia en el que puedan participar de forma voluntaria todas las universidades.

Las universidades desarrollan libremente sus SGC en base a su misión, visión y plan estratégico, tradición histórica y estructura interna, entre otros factores.

Los principales criterios que las universidades han utilizado para el desarrollo de los SGC son los siguientes:

- Optar por un diseño transversal de enfoque genérico, adecuado para todos sus centros (facultades y escuelas universitarias), teniendo en cuenta que los procedimientos específicos y los indicadores deben depender de cada uno de los centros. En esta situación, es la universidad la que se encarga de diseñar el SGC y cada centro recibe información del proceso, siendo su participación muy limitada.

- El centro elabora su SGC y la universidad se encarga de la coordinación, especialmente en relación a los elementos transversales y más centralizados (por ejemplo, en lo referido al personal académico, a los recursos materiales y servicios, etc.).

Programa AUDIT

\footnotetext{
${ }^{1}$ AUDIT ha sido diseñado por la "Axencia para a Calidade do Sistema Universitario de Galicia - ACSUG" (Agencia para la Calidad del Sistema Universitario de Galicia), la "Agencia Nacional de Evaluación de la Calidad y Acreditación - ANECA" y la "Agència per a la Qualitat del Sistema Universitari de Catalunya - AQU Catalunya" (Agencia para la Calidad del Sistema Universitario de Cataluña), posteriormente se incorporó la "Euskal Unibertsitate Sistemaren Kalitate Agentzia - UNIBASQ" (Agencia de Calidad del Sistema Universitario Vasco).
} 
AUDIT está desarrollado siguiendo las directrices de ENQA ${ }^{2}$ (Tabla 1) con el objetivo de cubrir todas las dimensiones y los grupos de interés involucrados en el proceso formativo:

- Política y procedimientos para la garantía de calidad.

- Aprobación, control y revisión periódica de programas y títulos.

- Evaluación de los estudiantes.

- Garantía de calidad del personal docente.

- Recursos de aprendizaje y apoyo al estudiante.

- Sistemas de información.

- Información pública.

Este programa lo presentan, oficialmente, las tres agencias a las universidades del SUE el 25 de junio de 2007, como una herramienta con dos objetivos básicos:

a. Facilitar a los centros orientaciones para el diseño, desarrollo e implementación de los SGC.

b. Poner en práctica procedimientos de reconocimiento del diseño y certificación de la implantación de los SGC en los centros.

La institución debe abordar, de modo progresivo, estas etapas, dejando evidencia documental del cumplimiento de cada una de ellas.

El Programa se estructura en 7 directrices, 8 en AQU Catalunya ${ }^{3}$ y 10 en ACSUG (Programa FIDES-AUDIT) $)^{4}$ :

- Cómo el Centro define su política y objetivos de calidad. La consolidación de la cultura de calidad ha de apoyarse en una política y objetivos de calidad de la formación conocidos y accesibles públicamente, además de ser revisados y mejorados periódicamente.

- Cómo el Centro garantiza la calidad de sus programas formativos. El mantenimiento y renovación de la oferta formativa ha de apoyarse en el desarrollo de metodologías para el diseño, la aprobación, la revisión y la mejora de los programas formativos y, en su caso, de la extinción de ellos.

- Cómo el Centro orienta sus enseñanzas a los estudiantes. Las acciones que se emprenden en el marco del programa formativo han de tener como finalidad favorecer el aprendizaje del estudiante.

- Cómo el Centro garantiza y mejora la calidad de su personal. La política de personal académico y de administración y servicios ${ }^{5}$ ha de ser definida e implementada con

2 European Association for Quality Assurance in Higher Education. Standards and guidelines for Quality Assurance in the European Higher Education Area.

${ }^{3}$ AQU Catalunya, a partir del proceso de metaevaluación llevado a cabo después de la primera convocatoria (2007), añadió una nueva directriz, "Aspectos Generales del sistema de garantía de calidad”, donde el centro debe identificar la interrelación entre los procesos definitivos, determinando el responsable de cada proceso, indicadores, gestión de la documentación del SGC y revisión global de implementación de las mejoras del mismo.

${ }^{4}$ ACSUG había presentado el Programa FIDES (Establecimiento del Sistema de Garantía de Calidad de Títulos Universitarios) a sus universidades, previo al Programa AUDIT, y las 10 directrices son resultado de la fusión de ambos programas. Se añadieron 3 directrices: Definición de los objetivos del plan de estudios, Definición de la política y los procedimientos de admisión y Diseño, aprobación e implementación de la planificación de la enseñanza.

${ }^{5}$ El personal de administración e servicios al que se refiere esta directriz es únicamente el personal relacionado con la formación universitaria: conserjes, secretarios, apoyo a la docencia (laboratorios, talleres, bibliotecas...) y cualquier otro que la universidad considere vinculado a la formación. 
las debidas garantías para asegurar su suficiencia numérica, competencia y cualificación.

- Cómo el Centro gestiona y mejora sus recursos materiales y servicios. El correcto diseño, gestión, revisión y mejora de los servicios y recursos materiales ${ }^{6}$ ha de estar asegurado para un adecuado desarrollo del aprendizaje de los estudiantes.

- Cómo el Centro analiza y tiene en cuenta los resultados. Los resultados han de ser recogidos y analizados para la revisión y mejora de la calidad de los programas formativos.

- Cómo el Centro publica la información sobre las titulaciones. Garantizar la publicación periódica de información actualizada y la rendición de cuentas relativas a los programas formativos impartidos y aspectos relacionados.

Para cada una de ellas se debe especificar el órgano responsable, describir cómo participan los diferentes grupos de interés, establecer los mecanismos para la medición, revisión y mejora de los procesos y desarrollar claramente los mecanismos para la información pública y rendición de cuentas a la sociedad.

A su vez, cada directriz se desglosa en una serie de elementos, algunos de los cuales están regulados a nivel nacional en el marco del diseño de los programas educativos.

Tabla 1. Relación entre los criterios $\mathrm{ESG}^{7}$ (y las directrices del programa AUDIT

\begin{tabular}{|l|l|}
\hline \multicolumn{1}{|c|}{ ESG } & \multicolumn{1}{c|}{ AUDIT } \\
\hline $\begin{array}{l}\text { 1.1 Política y procedimientos para la garantía de } \\
\text { calidad }\end{array}$ & $\begin{array}{l}\text { Directriz 1. Cómo el Centro define su política y } \\
\text { objetivos de calidad }\end{array}$ \\
\hline $\begin{array}{l}\text { 1.2. Aprobación, control y revisión periódica de } \\
\text { programas y títulos }\end{array}$ & $\begin{array}{l}\text { Directriz 2. Cómo el Centro garantiza la calidad } \\
\text { de sus programas formativos }\end{array}$ \\
\hline $\begin{array}{l}\text { 1.3. Evaluación de los estudiantes } \\
\text { 1.4. Garantía de calidad del personal docente }\end{array}$ & $\begin{array}{l}\text { Directriz 3. Cómo el Centro orienta sus } \\
\text { enseñanzas a los estudiantes }\end{array}$ \\
\hline $\begin{array}{l}\text { 1.5. Recursos de aprendizaje y apoyo al } \\
\text { estudiante }\end{array}$ & $\begin{array}{l}\text { Directriz 5. Cómo el Centro garantiza y mejora la } \\
\text { sus recursos materiales y servicios }\end{array}$ \\
\hline $\begin{array}{l}\text { 1.6. Sistemas de información } \\
\text { 1.7. Información pública }\end{array}$ & $\begin{array}{l}\text { Directriz 6. Cómo el Centro analiza y tiene en } \\
\text { cuenta los resultados }\end{array}$ \\
\hline
\end{tabular}

AUDIT incluye dos secuencias de actuación, tanto para las universidades como para las agencias. Las universidades diseñan sus SGC para implantarlos posteriormente y las

\footnotetext{
${ }^{6}$ Instalaciones (aulas, salas de estudio, aulas de informática, laboratorios, salas de reunión, puestos de lectura en biblioteca,...) y equipamiento como material científico, técnico, asistencial y artístico.

${ }^{7}$ European Standards and Guidelines elaborados por ENQA.
} 
agencias evalúan estos diseños y, finalmente certifican, a través de una auditoría, la implantación de los mismos.

\section{Evaluación del diseño del SGC}

La fase de evaluación del diseño tiene una gran importancia ya que asegura la calidad del SGC propuesto por el centro/universidad, según las directrices de referencia establecidas por AUDIT, y es el paso previo a la posterior fase de implementación y certificación.

Las agencias participantes en el programa han elaborado un protocolo de evaluación, que pretende servir:

- Como herramienta de trabajo para los evaluadores, en su labor de valorar con el mayor nivel de objetividad y homogeneidad posible los SGC diseñados por los centros/universidades.

- Para ayudar a las universidades a conocer en detalle el proceso de evaluación, el protocolo de evaluación y los aspectos que serán tenidos en cuenta por los evaluadores, para poder actuar en consecuencia y reforzar aquellos aspectos que se consideran de especial significación en el diseño del SGC.

Las Agencias, durante el período 2008-2012, abrieron convocatorias anuales para que los centros de las universidades españolas presentasen la solicitud para participar en la misma.

Las actividades de evaluación se realizaron a través de comisiones de evaluadores formados por expertos externos al sistema universitario del que depende la universidad, excepto los vocales profesionales y estudiantes que son de la propia Comunidad Autónoma a la que pertenece la misma. Estas comisiones están compuestas por el número de miembros necesario para realizar la evaluación con garantía.

La selección de cada uno de los miembros de las comisiones se realiza a partir de la base de datos de evaluadores externos de la que dispone cada Agencia, siguiendo los criterios y directrices establecidos por las mismas.

Cada uno de los miembros de la comisión analiza de forma individual la documentación del sistema, evaluando la conformidad con los criterios del programa. Posteriormente se realiza una reunión de consenso para comparar las evaluaciones y llegar a un común acuerdo. A continuación se elabora un informe provisional que se remite a la universidad. Tras el periodo de alegaciones se elabora un informe final. A partir de este momento el centro debe comenzar la implantación de su SGC.

La valoración global del informe provisional puede ser: positiva, positiva condicionada a la elaboración de un plan de mejoras o negativa, e incluye tres apartados: justificación de la valoración global, fortalezas y puntos débiles-propuestas de mejora.

La valoración global del informe final puede ser positiva o negativa, incluyendo tres apartados: justificación de la valoración global, fortalezas y sugerencias para la implantación del SGC y su futuro seguimiento y certificación.

La evaluación del diseño del SGC tiene una validez indefinida.

\section{Certificación de la implantación del SGC}

El proceso de certificación de la implantación de los SGC se ha presentado a las universidades en 2012 y ha sido desarrollado por ACSUG, ANECA y UNIBASQ, con Ios objetivos de: 
- Obtener un certificado de calidad otorgado por las Agencias, como forma de reconocimiento y difusión pública del nivel de madurez alcanzado en la implantación del SGC.

- Facilitar a las Universidades las orientaciones que ayuden a evaluar internamente el grado de implantación del SGC, con el fin de mejorarlo.

- Proporcionar a los equipos de auditores externos las orientaciones y documentos que les permitan desarrollar de forma ordenada, objetiva y homogénea su trabajo.

La certificación de la implantación de los SGC busca como objetivos complementarios a los ya descritos, que las universidades puedan economizar recursos y tiempo en la evaluación de sus títulos.

La participación de las universidades en la etapa de certificación de la implantación de los SGC continúa teniendo un carácter voluntario. Las agencias instan a las universidades a asumir el reto de obtener un distintivo de calidad que demuestre su implicación con la mejora continua y demostrar que están en disposición de hacer públicos sus resultados ante los diferentes grupos de interés de acuerdo a los objetivos y principios expresados en los Criterios y Directrices de garantía de calidad del EEES.

La implantación de un SGC en un centro requiere de una sistematización y estructuración de los procesos de garantía de calidad existentes y contempla, entre otros procesos claves, la planificación de la oferta formativa, la evaluación y revisión de su desarrollo, así como la toma de decisiones para la mejora de la formación.

Durante el proceso de certificación, y como objetivo general, los auditores externos determinarán si el sistema implantado sigue cumpliendo las directrices definidas en el Programa AUDIT. Debe ponerse de manifiesto que el SGC implantado permite que el ciclo de mejora continua resulte eficaz y operativo. Finalmente se comprobará que el sistema se mantiene de manera adecuada en el tiempo.

Las agencias abren convocatorias anuales ${ }^{8}$ para que las universidades presenten la solicitud para participar en el proceso de certificación de la implantación del SGC. Dicho proceso se realiza a través de una auditoría externa. Para elegir los miembros de cada equipo, se tendrá en cuenta el origen geográfico profesional de las personas que actuarán como auditores, de manera que no se seleccionarán personas que trabajen en universidades que pertenezcan a la Comunidad Autónoma en la que se encuentra la universidad, excepto los vocales profesionales y estudiantes.

La selección de cada uno de los miembros del equipo auditor se realiza a partir de la base de datos de evaluadores externos de la que dispone cada Agencia, siguiendo los criterios y directrices establecidos por las mismas.

Cada uno de los miembros del equipo realiza, de forma individual, una revisión preliminar de la documentación del SGC y posteriormente, como norma general, se desarrolla una auditoría de tipo presencial en los centros. Para recabar la información, además de la revisión documental, se realizan audiencias con el equipo directivo y la comisión de garantía de calidad (CGC) y, si el equipo auditor lo considera necesario, se realizarán audiencias con otros grupos de interés (coordinadores de títulos, unidades técnicas, profesorados, alumnado, egresados, empleadores) así como una visita a las instalaciones. Al finalizar la auditoría se elabora un informe provisional que se remite a la universidad. Tras el periodo de alegaciones se elabora un informe final.

\footnotetext{
${ }^{8}$ ANECA tiene una convocatoria abierta permanentemente.
} 
El proceso evalúa la conformidad con los criterios del programa y el informe puede incluir no conformidades de tipo mayor ${ }^{9}$ o menor $^{10}$. En caso de que el informe de auditoría incluya alguna no conformidad, el centro debe elaborar un plan de acciones de mejora que será remitido a la agencia y revisado por el equipo auditor antes de la elaboración del informe final y decisión relativa a la certificación del SGC.

La valoración global del informe provisional puede ser: favorable, condicionado a la elaboración de un plan de mejoras o desfavorable, e incluye cuatro apartados: justificación del resultado, detalle de las no conformidades existentes, fortalezas detectadas y oportunidades para la mejora.

La valoración global del informe final puede ser favorable o desfavorable e incluye cuatro apartados: justificación del resultado, detalle de las no conformidades existentes, fortalezas detectadas y oportunidades para la mejora.

El certificado concedido por las agencias tendrá una validez de cuatro años prorrogable por idénticos periodos, tras superar una auditoría de renovación (caso de ANECA y UNIBASQ) y de seis años en el caso de ACSUG (cada año, el centro, debe enviar a la Agencia el plan de acciones de mejora, a los tres años se realizará una auditoría de seguimiento y al cabo de los seis años se realizará una auditoría de renovación del certificado).

\section{Resultados}

Los resultados globales de la evaluación del diseño de los SGC que se presentan ${ }^{11}$ se corresponden con evaluaciones favorables. Aquellos centros que en alguna convocatoria obtuvieron informe negativo se han presentado en siguientes convocatorias obteniendo informe positivo.

El SUE ${ }^{12}$ está formado por 77 universidades, de las cuales 50 son públicas y 27 privadas (Gráfico 1).

\footnotetext{
${ }^{9}$ No existe evidencia, o ésta es inadecuada, acerca del cumplimiento de la cuestión planteada por el elemento.

${ }^{10}$ Se aportan evidencias puntuales de la existencia de contenidos en la documentación y en el uso del SGC relacionados con la cuestión planteada por el elemento, pero no se ajustan en su totalidad a las directrices establecidas en el Programa AUDIT y/o no están correctamente definidos o no se ejecutan de manera sistemática.

${ }^{11}$ Fuente: ACSUG, ANECA, AQU Catalunya y UNIBASQ.

${ }^{12}$ Fuente: Ministerio de Educación, Cultura y Deporte. Gobierno de España.
} 


\section{SISTEMA UNIVERSITARIO ESPAÑOL}

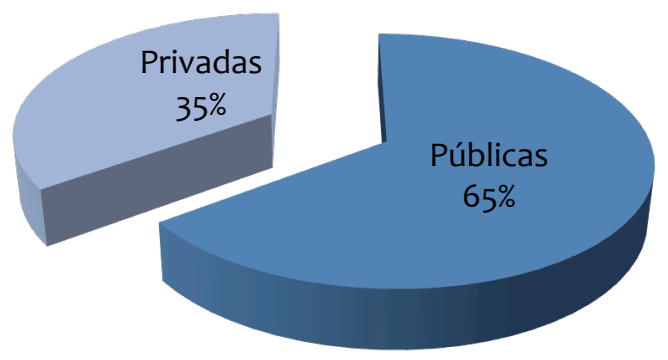

Gráfico 1. Universidades públicas y privadas del SUE

Es muy destacable el elevado grado de implicación y participación de las universidades en AUDIT. Entre 2007 y 2013 han participado en el proceso de evaluación del diseño de los SGC 62 universidades españolas, lo que representa el $80,5 \%$ del total de universidades del SUE. 20 de ellas son privadas (32\%) y 42 públicas (68\%) (Gráfico 2 ).

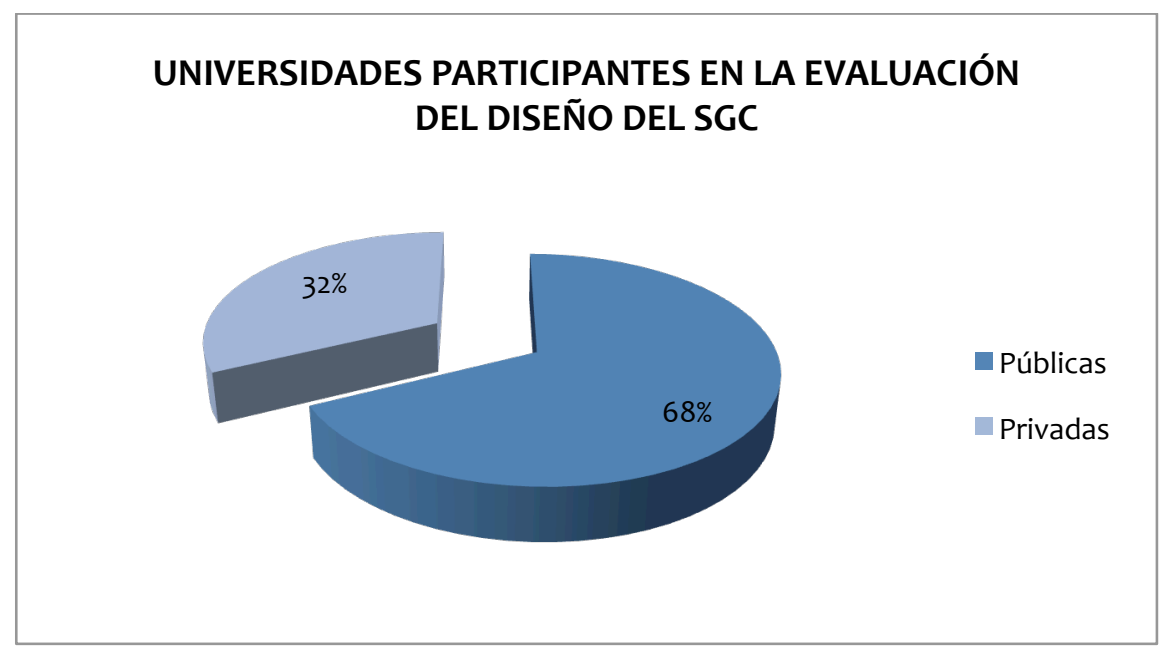

Gráfico 2. Universidades participantes en el Programa AUDIT

De las 62 universidades participantes, la mayoría han optado por desarrollar un SGC de centro, teniendo evaluados favorablemente el diseño de todos sus centros 21 universidades (34\%) (Gráfico 3). 


\section{Evaluación del diseño del SGC}

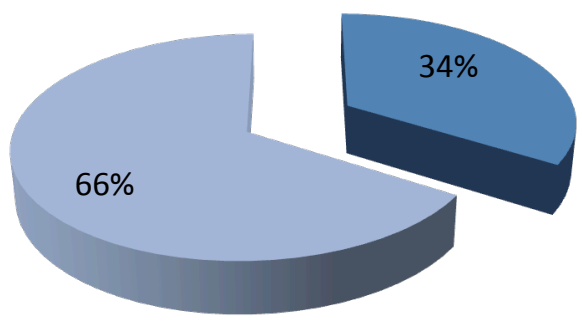

Todos los centros

Uno o varios centros

Gráfico 3. Evaluación por centro del SGC

La distribución de las 62 universidades participantes en AUDIT por agencias es la siguiente (Tabla 2):

Tabla 2. Distribución de Universidades por Agencias

\begin{tabular}{|c|c|c|c|c|c|c|}
\hline \multirow{2}{*}{\multicolumn{2}{|c|}{$\begin{array}{l}\text { UNIVERSIDADES TERRITORIO } \\
\text { AGENCIAS }\end{array}$}} & \multicolumn{5}{|c|}{ AGENCIAS } \\
\hline & & \multirow{2}{*}{$\frac{\text { ACSUG }}{3}$} & \multirow{2}{*}{ ANECA } & \multirow{2}{*}{$\begin{array}{c}\text { AQU Catalunya } \\
7\end{array}$} & \multirow{2}{*}{$\begin{array}{c}\text { UNIBASQ } \\
1\end{array}$} & \multirow{2}{*}{$\begin{array}{c}\text { TOTAL } \\
42\end{array}$} \\
\hline \multirow{3}{*}{$\begin{array}{c}\text { UNIVERSIDADES } \\
\text { PARTICIPANTES } \\
\text { AUDIT }\end{array}$} & PÚBLICAS & & & & & \\
\hline & PRIVADAS & 0 & 13 & 5 & 2 & 20 \\
\hline & TOTAL & 3 & 44 & 12 & 3 & 62 \\
\hline \multirow{3}{*}{$\begin{array}{l}\text { UNIVERSIDADES } \\
\text { TOTALES }\end{array}$} & PÚBLICAS & 3 & 39 & 7 & 1 & 50 \\
\hline & PRIVADAS & 0 & 20 & 5 & 2 & 27 \\
\hline & TOTAL & 3 & 59 & 12 & 3 & 77 \\
\hline
\end{tabular}

EL gráfico 4 muestra la distribución de las universidades del SUE por territorio de las Agencias. El número elevado de universidades que corresponden al territorio ANECA es debido a que, aun existiendo agencias autonómicas, estas no se han involucrado en el programa AUDIT. 


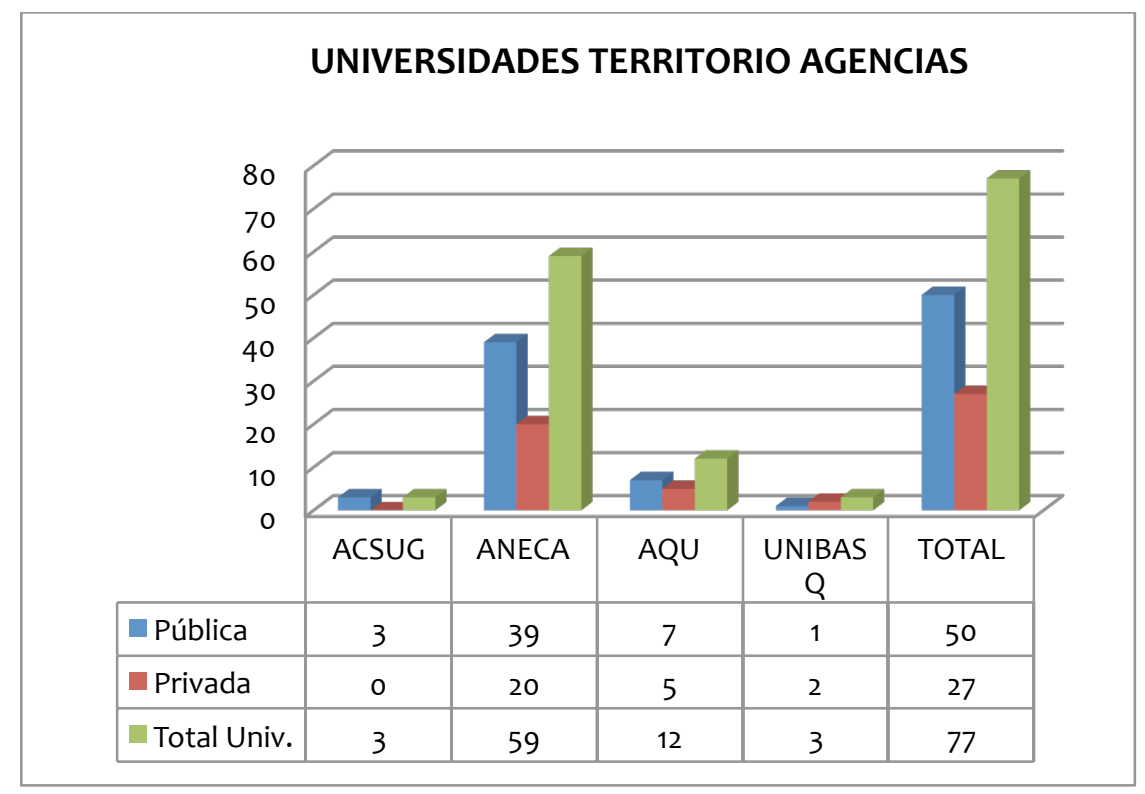

Gráfico 4. Distribución de universidades por Agencia

En ACSUG, AQU Catalunya y UNIBASQ ha participado el $100 \%$ de sus universidades en la evaluación del diseño de los SGC. En ANECA participó el 74,6\% de sus universidades (Gráfico 5).

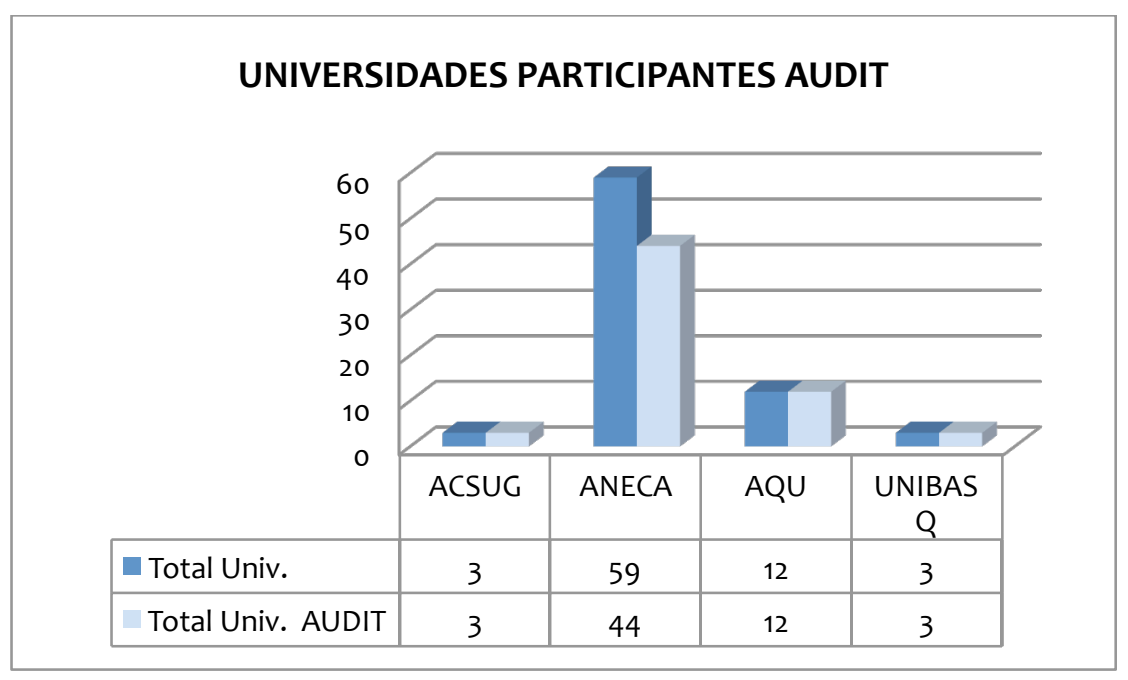

Gráfico5. Universidades participantes en AUDIT por Agencias

Todas las universidades de ACSUG y el 59\% de las universidades de ANECA tienen evaluado el diseño del SGC en todos sus centros, en cambio la totalidad de las universidades de AQU Catalunya y UNIBASQ tienen evaluado el diseño del SGC en alguno de sus centros (Gráfico 6). 


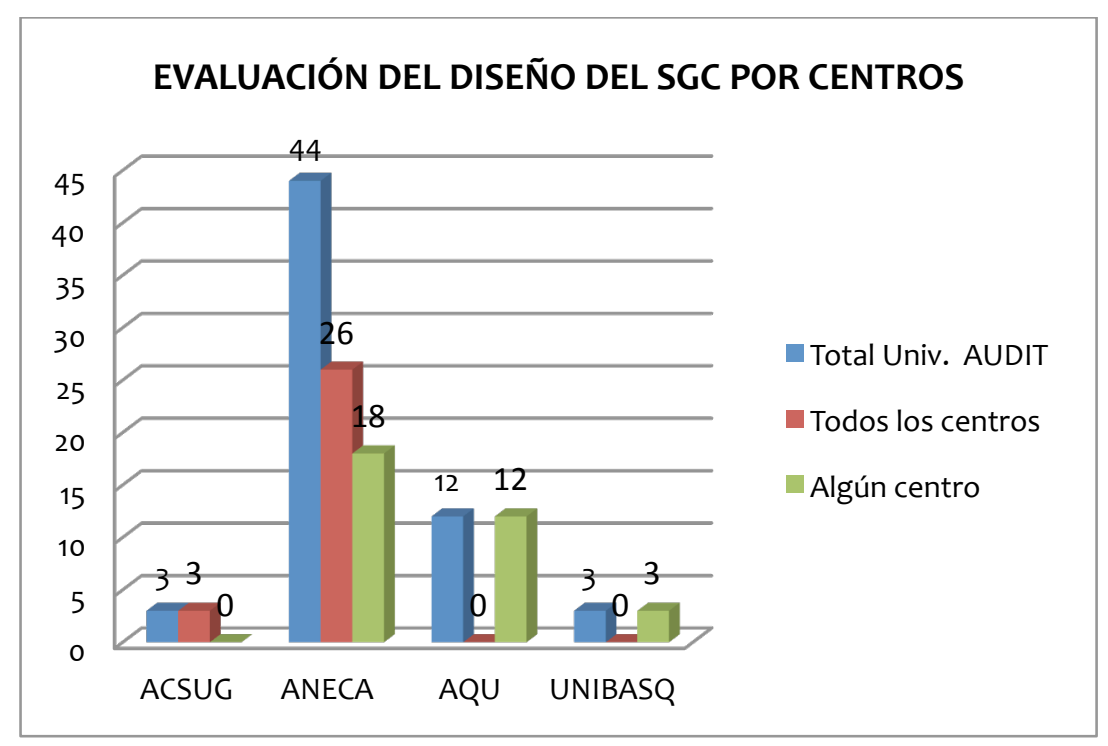

Gráfico 6. Evaluación del diseño en todos o alguno de los centro de la universidad por agencia

El gráfico 7 expresa el \% de universidades públicas y privadas participantes en AUDIT en cada agencia, en relación con el total de universidades del SUE participantes en dicho programa.

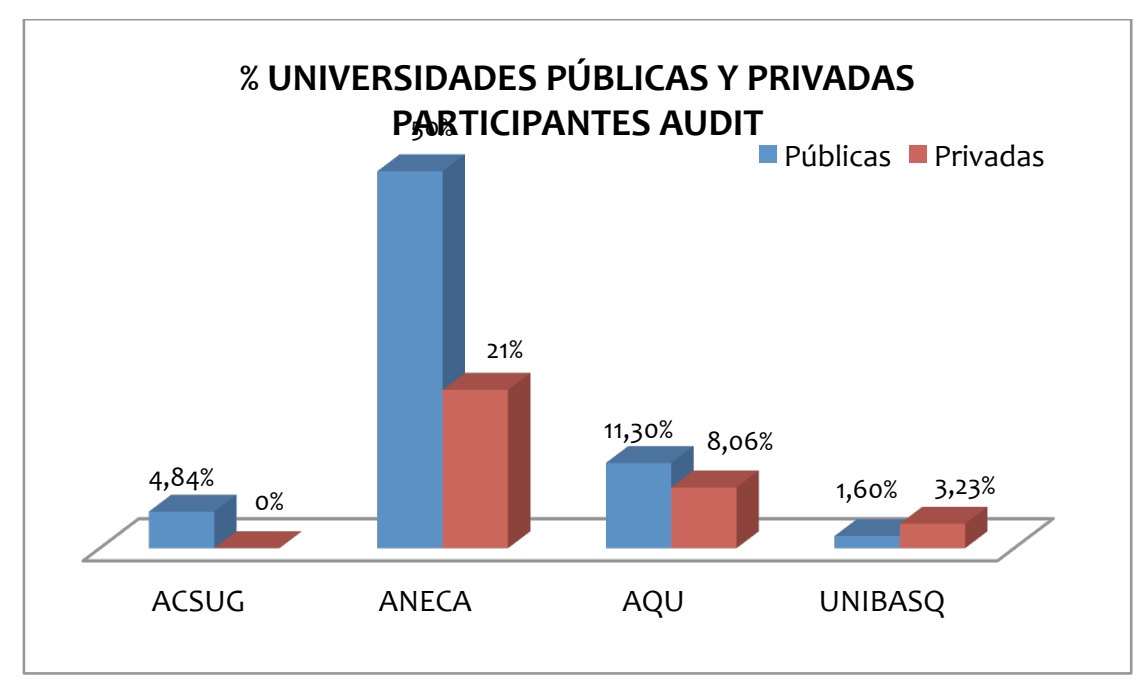

Gráfico 7. \% de universidades públicas y privadas participantes en AUDIT en cada Agencia

En 2013 se desarrolló una fase piloto de certificación de la implantación de los SGC, habiendo participado 12 universidades con 20 centros (Tabla 3).

Tabla 3. Universidades/centros participantes en el piloto de certificación

\begin{tabular}{|c|c|c|}
\hline AGENCIAS & UNIVERSIDADES & CENTROS \\
\hline ACSUG & 3 & 5 \\
\hline
\end{tabular}




\begin{tabular}{|c|c|c|}
\hline ANECA & 7 & 13 \\
\hline UNIBASQ & 2 & 2 \\
\hline TOTAL & 12 & 20 \\
\hline
\end{tabular}

De las 12 universidades han obtenido un informe favorable 8 de ellas: 2 de ACSUG, 4 de ANECA y 2 de UNIBASQ. 6 de las cuales son públicas y 2 privadas. De los 20 centros, han obtenido informe favorable 11: 4 de ACSUG, 5 de ANECA y 2 de UNIBASQ ${ }^{13}$.

A finales de 2013 se abre la primera convocatoria oficial ${ }^{14}$, habiendo realizado, en el primer semestre de 2014, visita a:

- ACSUG: 2 universidades, 12 centros.

- UNIBASQ: 2 universidades, 3 centros.

- ANECA: información no disponible en la web.

ACSUG (julio de 2014) ha enviado los informes provisionales estando a la espera de recibir las alegaciones y plan de mejoras, para emitir el informe final.

\section{Conclusiones}

Las agencias de evaluación han jugado un triple papel, han dado orientaciones y apoyo a las universidades para el diseño de los SGC; han evaluado los diseños presentados por las universidades y, actualmente, se está procediendo a la certificación de la implantación de los mismos.

Al comenzar a diseñar los SCC se han creado, en las universidades y/o centros, grupos de trabajo. Esto ha provocado el fomento de la cultura de calidad, así como la actualización y desarrollo de iniciativas sistemáticas de trabajo y mejora continua. Por ello, a medida que avanzaban las convocatorias, los diseños de los SGC eran más completos, lo que ha supuesto un aumento de las fortalezas y una disminución gradual de las debilidades y oportunidades para la mejora.

Al realizar las evaluaciones, las agencias han detectado, una serie de puntos débiles y fuertes que se repetían en el diseño del SGC de un gran número de universidades/centros, que se recogen en la tabla 4.

Tabla 4. Puntos débiles y puntos fuertes detectados en la fase de evaluación del diseño del SGC

\begin{tabular}{|c|c|}
\hline PUNTOS DÉBILES & PUNTOS FUERTES \\
\hline $\begin{array}{l}\text { - Indefinición del alcance del SGC. } \\
\text { - Documentación del SGC poco } \\
\text { contextualizada al centro. Esto se ha } \\
\text { detectado, en mayor medida, en los SGC } \\
\text { desarrollados por la universidad. } \\
\text { - Documentación del SGC muy amplia, }\end{array}$ & $\begin{array}{l}\text { - Disponer de una aplicación informática } \\
\text { que permite registrar y controlar las } \\
\text { versiones de la documentación del SGC. } \\
\text { - Definición de los objetivos de calidad del } \\
\text { centro teniendo en cuenta las } \\
\text { necesidades de los grupos de interés. }\end{array}$ \\
\hline
\end{tabular}

\footnotetext{
${ }^{13}$ Fuente: Páginas web de ACSUG, ANECA y UNIBASQ.

${ }^{14}$ ANECA tiene convocatoria abierta permanentemente.
} 


\begin{tabular}{|c|c|}
\hline $\begin{array}{l}\text { incluso incluyendo acciones que no se } \\
\text { realizan en el centro. } \\
\text { Especificación deficiente de las } \\
\text { responsabilidades de todos los } \\
\text { implicados en el SGC. } \\
\text { Definición imprecisa de los objetivos de } \\
\text { calidad, objetivos plurianuales, lo que } \\
\text { provoca que sean difícilmente medibles. } \\
\text { Definición incompleta de las funciones y } \\
\text { composición de los órganos } \\
\text { responsables de la gestión del SGC. } \\
\text { Desarrollo incompleto } \\
\text { procedimientos específicos para la } \\
\text { rendición de cuentas y para la toma de } \\
\text { decisiones relacionadas con el SGC. } \\
\text { Definición poco clara del método } \\
\text { operativo para llevar a cabo la gestión } \\
\text { del procedimiento de la inserción laboral. } \\
\text { Definición poco clara de cómo se } \\
\text { utilizarán los análisis de los resultados } \\
\text { para implementar mejoras en los títulos } \\
\text { que se imparten en el centro. }\end{array}$ & $\begin{array}{l}\text { - Implantación sólida, en la mayoría de los } \\
\text { centros, de cultura de calidad y mejora } \\
\text { continua. } \\
\text { Definición clara de los procedimientos que } \\
\text { garantizan la calidad de los programas } \\
\text { formativos. } \\
\text { - Definición clara de los procedimientos de } \\
\text { orientación de las enseñanzas a los } \\
\text { estudiantes } \\
\text { Definición clara de los procedimientos que } \\
\text { garantizan la calidad de los recursos } \\
\text { materiales y servicios. } \\
\text { La rendición de cuentas a los grupos de } \\
\text { interés externos mediante la publicación } \\
\text { periódica de la Memoria de } \\
\text { Responsabilidad Social. }\end{array}$ \\
\hline
\end{tabular}

En la tabla 5, se incluyen los puntos débiles y puntos fuertes que se repiten en la mayoría de los informes de las universidades/centros en la fase de certificación de la implantación de los SGC.

Tabla 5. Puntos débiles y puntos fuertes detectados en la fase piloto y primera convocatoria de la certificación de la implantación de los SGC

\begin{tabular}{|c|c|}
\hline PUN & PUNTOS FUERTES \\
\hline $\begin{array}{l}\text { - No se realiza la revisión anual con el } \\
\text { objetivo de mejorar y/o simplificar la } \\
\text { documentación del SGC, tal como se } \\
\text { establecía en el diseño del mismo. } \\
\text { Definición de objetivos de calidad poco } \\
\text { concretos, algunos inalcanzables, otros } \\
\text { de difícil medición y en general poco } \\
\text { motivadores. } \\
\text { - No se recoge información suficiente } \\
\text { sobre las necesidades de formación y } \\
\text { resultados de evaluación del personal } \\
\text { académico. } \\
\text { No se evidencia claramente que se } \\
\text { analice la información disponible ni que } \\
\text { se establezcan acciones de mejora } \\
\text { respecto a la satisfacción de los usuarios } \\
\text { con los servicios del centro. } \\
\text { No se evidencia claramente que se } \\
\text { realicen acciones para la recogida de } \\
\text { información, análisis y mejora respecto a } \\
\text { la satisfacción de los empleadores. }\end{array}$ & 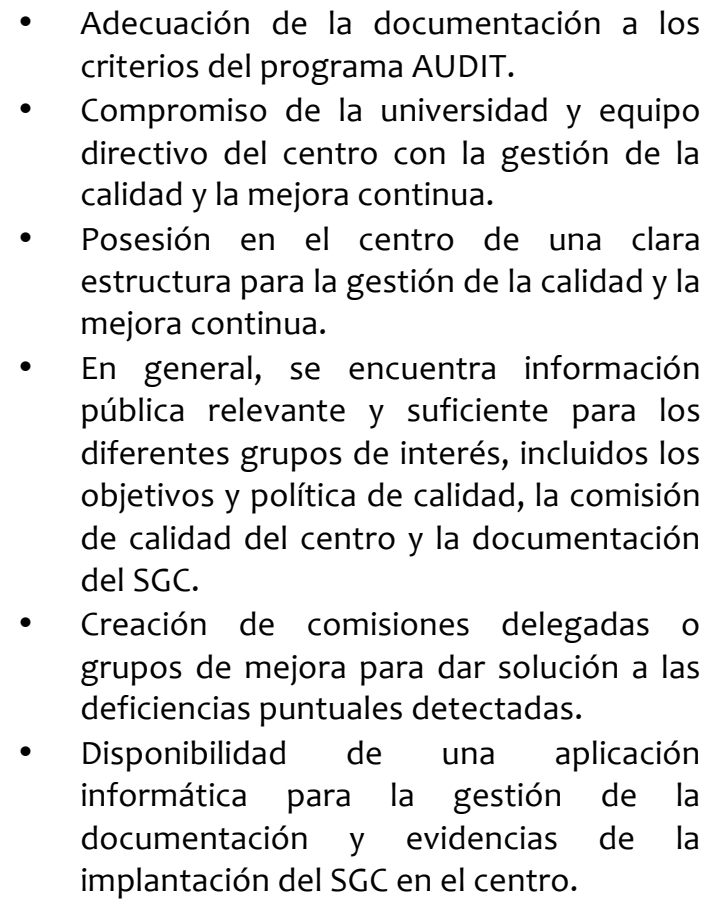 \\
\hline
\end{tabular}


- En algunos procedimientos no se completa el ciclo de información, análisis y mejora.

- No retorna la información a los interesados tras el análisis de la satisfacción de los diferentes grupos de interés implicados.

En ACSUG, coincidiendo con la primera convocatoria oficial de certificación de la implantación de los SGC (finales de 2013) se abría la convocatoria piloto para participar en la renovación de la acreditación de títulos oficiales, realizando en dos centros de una universidad gallega, la visita para la renovación de la acreditación y certificación de la implantación del SGC de forma simultánea.

De estas visitas se puede concluir que aquellos centros que tienen implantado el SGC muestran mayor facilidad para diseñar nuevos títulos, una capacidad adecuada para implantarlos, una madurez en el proceso de seguimiento de los mismos y demuestran una gran seguridad y garantía en la renovación de la acreditación de sus títulos. Esto implica que ambos centros tienen implantado eficiente y eficazmente un procedimiento de obtención de resultados, de análisis y toma de decisiones sobre estos, lo que facilita la mejora continua de los títulos que en ellos se imparten.

\section{Referencias}

Real Decreto 1393/2007, de 29 de octubre, por el que se establece la ordenación de las enseñanzas universitarias oficiales (texto consolidado).

Ley Orgánica 6/2001, de 21 de diciembre, de Universidades (texto consolidado).

ACSUG (http://www.acsug.es/es/centros/fidesaudit):

- Guía Programa FIDES (2007)

- Guía para el diseño de Sistemas de Garantía Interna de la Calidad de la formación universitaria (2007)

- Directrices, definición y documentación de los Sistemas de Garantía Interna de Calidad de la formación universitaria (2009)

- Protocolo de evaluación externa del diseño del Sistema de Garantía Interna de Calidad (2009)

- Herramientas para el diagnóstico (2007)

- Guía Certificación AUDIT-ACSUG (2013)

- Guía de evaluación para la renovación de la acreditación de títulos oficiales de grado y máster (2013)

ANECA (http://www.aneca.es/Programas/AUDIT/Fase-de-diseno-de-los-SGIC):

- Herramientas para el diagnóstico

- Directrices para el diseño de los Sistemas de Garantía Interna de Calidad de la formación universitaria

- Guía para el diseño de Sistemas de Garantía Interna de la Calidad de la formación universitaria

- Guía del Modelo de Certificación 
AQU Catalunya (http://www.aqu.cat/universitats/audit/index_es.html\#.U7FRi7F7Qbc):

- Guía para el diseño de Sistemas de Garantía Interna de Calidad de la formación Universitaria (2007)

- Directrices, definición y documentación de Sistemas de Garantía Interna de Calidad de la formación universitaria (2010)

- Herramientas para el Diagnóstico en la implantación de Sistemas de Garantía Interna de Calidad de la formación universitaria (2007)

- Guía de evaluación del diseño de Sistemas de Garantía Interna de Calidad de la formación universitaria (2010)

UNIBASQ (http://www.unibasq.org/es/programas/evaluacion-institucional/audit/):

- Guía para la Certificación de la implantación de Sistemas de Garantía de Calidad 


\section{Autores}

Dra. Ma Paula Ríos de Deus

Técnico de Programas de la Agencia para la Calidad del Sistema Universitario de Galicia. Experiencia en evaluación institucional y Gestión de la Calidad Universitaria desde 2001. Responsable del Programa AUDIT en ACSUG. Miembro de las comisiones de evaluación de verificación, seguimiento, modificaciones y renovación de la acreditación de títulos oficiales de ACSUG. Miembro de las comisiones de evaluación del diseño y certificación de la implantación de los SGC de ACSUG. Miembro de las comisiones de evaluación internacional de ACSUG 